\title{
DESVANTAGEM VOCAL EM CANTORES DE IGREJA
}

\section{Vocal handicap of church singers}

\author{
Tatiane Prestes ${ }^{(1)}$, Eliane Cristina Pereira ${ }^{(2)}$, Denise lenk Bail (3) , Ana Paula Dassie-Leite ${ }^{(4)}$
}

\section{RESUMO}

Objetivo: avaliar a desvantagem vocal de cantores amadores de coros de igreja. Método: participaram 42 cantores de coros amadores de igrejas, sendo 20 homens e 22 mulheres, com idades entre 18 e 59 anos. Todos responderam a um questionário contendo perguntas sobre autopercepção vocal e práticas de canto, e ao protocolo Índice de Desvantagem para o Canto Moderno (IDCM), composto por 30 questões referentes às subescalas incapacidade, desvantagem e defeito. Foi realizada triagem perceptivo-auditiva para classificação das vozes em adaptadas ou alteradas e mensuração dos graus de alteração. Resultados: a pontuação total média obtida no IDCM foi 23 pontos. Os maiores escores foram obtidos na subescala "defeito" $(10,9)$, seguido por "incapacidade" $(7,6)$ e "desvantagem" $(4,5)$, com diferença entre elas $(p=0,001)$. Cantores que nunca realizaram aula de canto apresentaram maiores escores no domínio "desvantagem" ( $p=0,003)$. À medida que o escore total do IDCM aumentou, a nota atribuída pelo cantor em relação à própria voz diminuiu $(p=0,046)$. Participantes com qualidade vocal alterada apresentaram maiores escores nas subescalas incapacidade e desvantagem e no domínio total do IDCM quando comparados aos que apresentavam qualidade vocal adaptada ( $p=0,012, p=0,049$ e $p=0,015$, respectivamente). Além disso, quanto maior o grau de alteração vocal, maiores foram os escores referentes à subescala incapacidade $(p=0,022)$. Conclusão: cantores de igreja apresentam desvantagem vocal importante. Quando apresentam alterações vocais, esta desvantagem é ainda maior. Quanto maior o grau de alteração vocal, maiores as limitações referentes à voz cantada. Aulas de canto parecem minimizar a desvantagem vocal nessa população.

DESCRITORES: Voz; Distúrbios da Voz; Hábitos; Música

\section{INTRODUÇÃO}

A arte de cantar exige o envolvimento de vários recursos do aparelho fonador. Os ajustes necessários para o canto dependem das exigências impostas pelo estilo, tipo de música e interpretação feita pelo cantor ${ }^{1}$. Algumas destas características

(1) Acadêmica do curso de Fonoaudiologia da Universidade Estadual do Centro-Oeste - UNICENTRO, Irati/PR.

(2) Fonoaudióloga da Prefeitura Municipal de Prudentópolis/ PR; Mestre em Distúrbios da Comunicação pela Universidade Tuiuti do Paraná.

(3) Fonoaudióloga; Professora Colaboradora da Universidade Estadual do Centro-Oeste - UNICENTRO, Irati/PR; Mestre em Distúrbios da Comunicação pela Universidade Tuiuti do Paraná.

(4) Fonoaudióloga; Professora Assistente A do Departamento de Fonoaudiologia da Universidade Estadual do Centro Oeste - UNICENTRO, Irati/PR; Mestre em Saúde da Criança e do Adolescente pela Universidade Estadual de Campinas - UNICAMP.

Conflito de interesses: inexistente podem fazer com que o cantor tenha ajustes vocais inadequados. Por isso, para que cada estilo de canto seja executado com o menor prejuízo possível ao aparato vocal do indivíduo, é importante o auxílio do fonoaudiólogo ${ }^{2}$. Os cantores, que antes dependiam exclusivamente de orientações de professores de canto, hoje podem buscar por trabalho de aprimoramento vocal desenvolvido por profissionais da Fonoaudiologia.

Atualmente, a maior parte das igrejas possui grupos vocais, coros e cantores solistas e essa população necessita de orientações fonoaudiológicas quanto à saúde vocal ${ }^{3}$. Em geral, esses cantores são amadores e desenvolvem essa atividade por satisfação pessoal. Por não terem preparação específica, muitos deles têm queixas referentes à saúde vocal $^{4}$. As principais delas referem-se a dificuldades para atingir as notas agudas ou graves, falta de ar para terminar as frases musicais, sintomas como dor laríngea e sensação de garganta seca, voz fraca ou forte demais para o canto e falta de controle quanto à afinação ${ }^{5,6}$. Essas alterações 
podem ser resultantes de métodos empíricos, falta de conhecimentos sobre a produção vocal, classificação vocal errônea, uso da voz de maneira incorreta e falta de treinamento vocal no que se refere às técnicas de aquecimento e desaquecimento ${ }^{1 .} \mathrm{O}$ fonoaudiólogo é o profissional que pode intervir em vários desses quesitos e possibilitar uma melhor performance vocal do cantor.

Estudos mostram que alguns coralistas já receberam informações sobre respiração, aquecimento vocal, saúde da voz, técnicas de relaxamento, exercícios de voz e técnica vocal, dentre outros ${ }^{3}$. No entanto, outras pesquisas ainda têm apontado um grande índice de alterações vocais dentre essa população e, em contrapartida, um pequeno índice de cantores que procuram por orientações fonoaudiológicas ${ }^{7}$.

Considerando as investigações fonoaudiológicas referente aos cantores de igreja, os profissionais têm buscado mensurar os resultados obtidos em pesquisas para melhor compreensão das necessidades vocais do público em questão. Uma das alternativas para isso é a utilização de instrumentos validados, traduzidos para a língua portuguesa e que vêm tendo grande aplicabilidade na clínica vocal, como por exemplo, o "Índice de desvantagem vocal para o canto moderno" (IDCM) ${ }^{8}$, que permite que a desvantagem vocal enfrentada por cantores seja mensurada. Este protocolo foi desenvolvido a partir do protocolo de Índice de Desvantagem Vocal (IDV) desenvolvido nos Estados Unidos e validado em quase 20 países, inclusive no Brasil| ${ }^{9}$.

O IDV usualmente é aplicado em indivíduos adultos com queixas vocais e avalia três diferentes aspectos: incapacidade, desvantagem e defeito vocal. Apesar de sua indiscutível validade, sua sensibilidade para avaliar cantores é pobre, uma vez que os fatores associados à percepção do indivíduo sobre sua desvantagem na voz cantada não são abordados neste protocolo. Dessa maneira, ele não contempla a consequência de uma disfonia na vida dos cantores. Por esse motivo, o foniatra italiano Franco Fussi o adaptou e a tradução feita para a língua portuguesa manteve essa adaptação.

O protocolo IDCM mostra-se muito eficaz para a população de cantores, e deve ser utilizado como auxílio por fonoaudiólogos, preparadores vocais e regentes na verificação de possíveis problemas vocais em cantores. Por meio de sua aplicação, já se pôde concluir que coralistas com queixa vocal autorrelatam maior desvantagem vocal do que coralistas sem problema de voz e não cantores. Além disso, sabe-se que cantores que apresentam problemas de natureza orgânica apresentaram maior desvantagem em relação aos demais ${ }^{8}$.
O IDCM já foi utilizado para avaliar a desvantagem vocal de coralistas amadores de canto moderno ${ }^{8-10}$. Os autores concluíram que, não havendo queixa vocal, cantores amadores relatam maior desvantagem em relação a cantores eruditos profissionais. Atribui-se tal diferença ao fato de cantores amadores possuírem menor preparo quanto a uma condição vocal estável, ausência de treinamento de voz específico e menor conhecimento da fisiologia vocal.

Considerando as necessidades que ainda existem quanto à caracterização e reflexão sobre cantores, o objetivo geral deste trabalho foi avaliar a desvantagem vocal de cantores amadores de coros de igreja, relacionando os índices de desvantagem vocal com as variáveis: sexo, naipe, faixa etária, tempo de canto, carga horária de canto semanal, utilização de voz profissional falada, autopercepção da voz, qualidade vocal e grau de alteração.

\section{MÉTODO}

Foi realizado um estudo transversal, de caráter descritivo/exploratório e natureza quantitativa. Participaram 42 cantores de coros de igrejas católicas e evangélicas localizadas na cidade de Guarapuava-PR, de faixa etária entre 18 e 59 anos (média de 39,7 anos), sendo 20 homens e 22 mulheres.

O tempo de canto referido pelos cantores variou entre 1 e 41 anos (média de 12,8 anos). Quanto ao naipe, 11 eram sopranos, 11 contraltos, 12 tenores e 8 baixos. Adotou-se como critério de inclusão cantar em coro de igreja há pelo menos um ano. Em contrapartida, foram excluídos indivíduos menores de 18 anos, maiores de 59 anos e que já haviam passado ou passam por acompanhamento fonoaudiológico devido a problemas vocais.

Inicialmente, todos os cantores responderam a um questionário, contendo os dados de identificação: nome, idade, sexo, profissão, igreja que frequenta, tipo de coro que participa (feminino, masculino ou misto), naipe que canta no coro (soprano, contralto, tenor, baixo), tempo de canto (em anos), carga horária de canto semanal, possível atividade extra com utilização da voz profissional falada, formação em canto e se fez ou participou de aula de canto. Além disso, o questionário continha uma questão sobre a autopercepção vocal, em que o cantor deveria atribuir uma nota à voz, de 0 a 10, sendo que 0 indicava desgostar totalmente de 10 gostar totalmente da própria voz.

Em seguida, foi aplicado o protocolo IDCM $^{8}$, composto por 30 questões, divididas em três subitens: incapacidade, desvantagem e defeito, que correspondem respectivamente aos domínios: funcional (exemplo: "Por causa do meu problema 
de voz sou forçado a limitar meu tempo de estudo/ ensaio"), emocional (exemplo: "Fico preocupado quando me pedem para repetir um vocalize ou uma frase musical") e orgânico (exemplo: "Tenho problemas com o controle da respiração para o canto") do IDV.

Os cantores foram orientados a assinalar o espaço específico em frente às afirmativas. As respostas foram assinaladas em uma escala de Likert de 5 pontos de acordo com a frequência de ocorrência das situações em questão: 0- nunca, 1- quase nunca, 2- às vezes, 3- quase sempre e 4- sempre. O IDCM apresenta quatro escores: incapacidade (funcional), desvantagem (emocional) e defeito (orgânico), cada um com valor máximo de 40 pontos, e o total, composto pelo somatório dos anteriores, com um escore máximo de 120 pontos. Quanto maior a pontuação, maior a percepção de desvantagem vocal do indivíduo.

Após o preenchimento do protocolo IDCM, foi realizada uma triagem vocal, por meio de análise perceptivo-auditiva da fala espontânea, eliciada por meio da pergunta "porque você gosta de cantar". A triagem foi realizada pelas próprias pesquisadoras e permitiu que a voz fosse classificada em "adaptada" ou "alterada", e que fosse determinado o grau de alteração (discreto, moderado ou intenso), em caso de voz alterada.

A pesquisa foi realizada respeitando-se a resolução 196/96 da Comissão Nacional de Ética em Pesquisa / CONEP. Não houve riscos para os sujeitos e todos os participantes assinaram o Termo de Consentimento Livre e Esclarecido (TCLE). A pesquisa foi aprovada pelo Comitê de Ética em Pesquisa da Universidade Estadual do Centro-Oeste (COMEP-I), sob número 008/2010.

Após a coleta, os dados foram tabulados e analisados estatisticamente por meio de testes específicos. A comparação entre os domínios do IDCM foi feita utilizando o Teste de Friedmann. Foram utilizados os testes de Mann-Whitney e Kruskall Wallis para comparar os índices obtidos no IDCM com as variáveis categóricas: sexo (feminino ou masculino), naipe (soprano, contralto, tenor ou baixo), utilização da voz profissional falada (sim ou não), realização prévia de aula de canto (sim ou não), qualidade vocal (adaptada ou alterada) e grau de alteração (discreto, moderado ou intenso). Além disso, o teste de Correlação de Spearmann foi utilizado para correlacionar os escores obtidos no IDCM com as demais variáveis contínuas: idade, nota referente à autopercepção vocal, tempo de canto, carga horária de canto semanal. Foi adotado nível de significância de 0,05 ou $5 \%$.

\section{RESULTADOS}

Os dados referentes à distribuição dos escores médios obtidos pelos cantores de igreja nos domínios incapacidade, desvantagem, defeito e total do protocolo IDCM podem ser observados na Tabela 1. Devido ao valor elevado do desvio padrão (esperado, pois se trata de avaliação subjetiva), o cálculo do valor de $\mathrm{p}$ foi realizado a partir da posição dos dados (mediana).

Tabela 1 - Distribuição e comparação entre os escores médios obtidos no protocolo IDCM

\begin{tabular}{ccccc}
\hline IDCM & Incapacidade & Desvantagem & Defeito & Total \\
\hline Média & 7,6 & 4,5 & 10,9 & 23,0 \\
Mediana & 6,0 & 3,0 & 10,0 & 20,0 \\
Desvio Padrão & 6,1 & 5,0 & 7,0 & 16,1 \\
\hline Valor de $p$ & \multicolumn{5}{c}{$<0,001^{*}$} \\
\hline
\end{tabular}

Teste Friedman; ${ }^{*} p<0,05$

Em relação ao protocolo IDCM, quanto maior a pontuação, maior será a desvantagem vocal autorreferida pelo indivíduo. A pontuação média total obtida foi de 23 pontos. Houve diferença estatisticamente significante na comparação entre os escores dos três domínios do IDCM $(p=0,001)$, sendo que o maior escore médio foi obtido na subescala "defeito" $(10,9)$, seguido pelos obtidos quanto à "incapacidade" $(7,6)$ e "desvantagem" $(4,5)$, respectivamente.
Foi realizada a comparação entre escores obtidos nas subescalas do protocolo IDCM com as variáveis categóricas: naipe, sexo, realização prévia de aula de canto e utilização da voz profissional falada (Tabela 2). Houve diferença estatisticamente significante somente na relação com a variável "realização de aula de canto" $(p=0,003)$, mostrando que pessoas que nunca realizaram aula de canto apresentaram maiores escores no domínio "desvantagem" (correspondente ao fator sócio-emocional do IDV). 
Tabela 2 - comparação entre escores obtidos no IDCM e as variáveis: NAIPE, sexo, realização de aula de canto e utilização da voz profissional falada

\begin{tabular}{|c|c|c|c|c|c|c|}
\hline IDCM & & & Média & Mediana & $\begin{array}{l}\text { Desvio } \\
\text { Padrão }\end{array}$ & Valor de $p$ \\
\hline \multirow{10}{*}{ Incapacidade } & \multirow{4}{*}{ Naipe ${ }^{\star * *}$} & Baixo & 8,1 & 6,5 & 6,4 & \multirow{4}{*}{0,808} \\
\hline & & Contralto & 5,4 & 7,0 & 3,0 & \\
\hline & & Soprano & 7,3 & 5,0 & 4,6 & \\
\hline & & Tenor & 9,8 & 7,5 & 8,6 & \\
\hline & \multirow{2}{*}{ Sexo** } & Feminino & 6,3 & 6,0 & 3,9 & \multirow{2}{*}{0,383} \\
\hline & & Masculino & 9,1 & 6,5 & 7,7 & \\
\hline & \multirow{2}{*}{ Aula de canto ${ }^{\star \star}$} & Não & 8,0 & 6,0 & 5,9 & \multirow{2}{*}{0,685} \\
\hline & & Sim & 7,2 & 6,0 & 6,4 & \\
\hline & \multirow{2}{*}{$\begin{array}{l}\text { Voz profissional } \\
\text { falada }\end{array}$} & Não & 7,8 & 6,0 & 6,2 & \multirow{2}{*}{0,960} \\
\hline & & Sim & 7,5 & 6,5 & 6,1 & \\
\hline \multirow{10}{*}{ Desvantagem } & \multirow{4}{*}{ Naipe ${ }^{\star * *}$} & Baixo & 4,1 & 1,5 & 6,0 & \multirow{4}{*}{0,096} \\
\hline & & Contralto & 2,0 & 2,0 & 1,8 & \\
\hline & & Soprano & 5,2 & 3,0 & 4,9 & \\
\hline & & Tenor & 6,4 & 3,5 & 5,8 & \\
\hline & \multirow{2}{*}{ Sexo** } & Feminino & 3,6 & 3,0 & 3,9 & \multirow{2}{*}{0,387} \\
\hline & & Masculino & 5,5 & 3,0 & 5,8 & \\
\hline & \multirow{2}{*}{ Aula de canto** } & Não & 6,5 & 3,0 & 5,8 & \multirow{2}{*}{$0,003^{*}$} \\
\hline & & Sim & 2,1 & 2,0 & 1,9 & \\
\hline & \multirow{2}{*}{$\begin{array}{c}\text { Voz profissional } \\
\text { falada }^{* *}\end{array}$} & Não & 5,2 & 3,0 & 6,0 & \multirow{2}{*}{0,859} \\
\hline & & Sim & 3,9 & 3,0 & 3,8 & \\
\hline \multirow{10}{*}{ Defeito } & \multirow{4}{*}{ Naipe $^{\star \star *}$} & Baixo & 10,6 & 7,5 & 9,0 & \multirow{4}{*}{0,948} \\
\hline & & Contralto & 10,0 & 10,0 & 4,3 & \\
\hline & & Soprano & 10,4 & 12,0 & 5,4 & \\
\hline & & Tenor & 12,3 & 9,5 & 9,3 & \\
\hline & \multirow{2}{*}{ Sexo** } & Feminino & 10,2 & 10,5 & 4,7 & \multirow{2}{*}{0,801} \\
\hline & & Masculino & 11,7 & 9,0 & 9,0 & \\
\hline & \multirow{2}{*}{ Aula de canto** } & Não & 12,0 & 11,0 & 7,3 & \multirow{2}{*}{0,215} \\
\hline & & Sim & 9,6 & 9,0 & 6,6 & \\
\hline & Voz profissional & Não & 10,7 & 9,0 & 7,8 & 0579 \\
\hline & falada** & Sim & 11,0 & 10,0 & 6,4 & $0,5 / 9$ \\
\hline & & Baixo & 22,9 & 14,0 & 20,9 & \\
\hline & Naine ${ }^{* \star *}$ & Contralto & 17,4 & 18,0 & 7,0 & 0667 \\
\hline & Nalpe & Soprano & 22,8 & 23,0 & 11,2 & $0,66 /$ \\
\hline & & Tenor & 28,3 & 20,5 & 21,7 & \\
\hline & Seyo** & Feminino & 20,1 & 20,0 & 9,5 & 0960 \\
\hline Iotal & sexo"n & Masculino & 26,2 & 18,5 & 21,0 & 0,960 \\
\hline & A ula do canto** & Não & 26,4 & 21,0 & 17,8 & 0100 \\
\hline & Aula de canto & Sim & 18,8 & 16,0 & 13,0 & 0,100 \\
\hline & Voz profissional & Não & 23,6 & 18,0 & 19,0 & 0734 \\
\hline & falada** & Sim & 22,5 & 20,5 & 13,4 & 0,734 \\
\hline
\end{tabular}

Testes **Mann-Whitney $e^{* * *}$ Kruskal-Wallis ${ }^{*} p<0,05$ 
A Tabela 3 mostra a correlação entre os escores obtidos no IDCM e as vaiáveis contínuas: idade, tempo de canto, carga horária de canto semanal e autoavaliação vocal. Observa-se diferença estatisticamente significante na correlação entre a subescala total do IDCM e a variável autoavaliação $\left(p=0,046^{*}\right)$. Tais variáveis apresentaram correlação negativa, ou seja, à medida que o escore total do IDCM aumentou, a nota atribuída pelo sujeito em relação à própria voz foi menor.

Tabela 3 - Correlação entre escores obtidos no IDCM e as variáveis idade, tempo de canto, carga horária de canto semanal e autoavaliação vocal

\begin{tabular}{cccccc}
\hline \multirow{2}{*}{ Incapacidade } & Idade & $\begin{array}{c}\text { Tempo de } \\
\text { canto }\end{array}$ & $\begin{array}{c}\text { C/H canto } \\
\text { semanal }\end{array}$ & Autoavaliação \\
& Corr & $8,5 \%$ & $5,9 \%$ & $5,6 \%$ & $-27,7 \%$ \\
& Valor de p & 0,594 & 0,711 & 0,726 & 0,080 \\
\hline \multirow{2}{*}{ Desvantagem } & Corr & $10,7 \%$ & $-12,5 \%$ & $11,0 \%$ & $-27,1 \%$ \\
& Valor de p & 0,502 & 0,430 & 0,488 & 0,087 \\
\hline \multirow{2}{*}{ Defeito } & Corr & $15,9 \%$ & $-13,3 \%$ & $-1,6 \%$ & $-27,2 \%$ \\
& Valor de p & 0,316 & 0,401 & 0,921 & 0,085 \\
\hline \multirow{2}{*}{ Total } & Corr & $14,8 \%$ & $-12,7 \%$ & $-0,2 \%$ & $-31,4 \%$ \\
& Valor de p & 0,348 & 0,424 & 0,989 & $0,046^{*}$ \\
\hline
\end{tabular}

Teste Correlação de Spearman ; * $p<0,05$

Foram comparados, ainda, os domínios obtidos pelos indivíduos no protocolo IDCM com os resultados da triagem vocal realizada pelas pesquisadoras no momento da coleta (Tabela 4). O domínio incapacidade apresentou diferença quanto à qualidade vocal e ao grau de alteração vocal. Verificou-se que os participantes com qualidade vocal adaptada autorreferiram menor incapacidade quando comparados aos que apresentam qualidade vocal alterada $(p=0,012)$. Além disso, quanto maior o grau de alteração vocal apresentada pelos participantes, maiores foram os escores referentes à incapacidade $(p=0,022)$. Houve diferença ainda, na comparação entre a subescala desvantagem e o domíniototalcomos dados dequalidadevocal. Assim, observa-se que indivíduos com qualidade vocal alterada apresentam maior desvantagem vocal $(p=0,049$ e $p=0,015$, respectivamente).

\section{DISCUSSÃO}

O conhecimento e a investigação sobre a desvantagem vocal referida pelos cantores amadores são fundamentais para a atuação de fonoaudiólogos junto a este público. Para isso, a utilização de protocolos, como o IDCM, mostra-se bastante sensível e eficaz ${ }^{10-12}$. A aplicação do IDCM fornece ao avaliador uma medida de autopercepção da gravidade do possível problema vocal enfrentado pelo cantor. Além disso, com o auxílio dos resultados, é possível conhecer as reais necessidades de cada cantor $^{13}$.

Neste estudo, a pontuação geral média obtida pelos cantores no protocolo IDCM foi de 23 prontos. Cantores que apresentaram vozes adaptadas tiveram escores médios totais de 19 pontos e os que apresentaram vozes alteradas tiveram escores médios em torno de 29 pontos. Tais dados corroboram resultados de estudo recente, que obteve média de 26,91 pontos para cantores com queixas vocais e 16,61 pontos para cantores sem queixas vocais ${ }^{8}$.

Considerando que o IDCM refere-se à adaptação do IDV para cantores, torna-se relevante a comparação dos resultados aqui obtidos com os dados recentemente publicados a partir da utilização do IDV. Para não cantores, estudo com o IDV apresenta escores médios totais obtidos por indivíduos disfônicos e não disfônicos. Em estudo sobre a comparação entre os protocolos de autoavaliação vocal validados no Brasil, obteve-se a informação de que indivíduos com vozes saudáveis apresentam escores totais de IDV próximos a 4,5 pontos ${ }^{14}$. Desta maneira, os resultados obtidos no presente estudo fazem-nos pensar que dificuldades vocais têm representado impacto considerável na performance e na vida de cantores, equiparando-os com indivíduos não cantores com alteração vocal comprovada.

Neste estudo, pode-se observar que a subescala defeito obteve as maiores médias de escores. 
Tabela 4 - comparação entre os escores médios obtidos no IDCM e os dados referentes à qualidade vocal e grau de alteração

\begin{tabular}{|c|c|c|c|c|c|c|}
\hline IDCM & & & Média & Mediana & $\begin{array}{l}\text { Desvio } \\
\text { Padrão }\end{array}$ & p-valor \\
\hline \multirow{5}{*}{ Incapacidade } & \multirow{3}{*}{$\mathrm{Grau}^{* * *}$} & Sem Alteração & 5,2 & 5,0 & 3,2 & \multirow{3}{*}{$0,022^{*}$} \\
\hline & & Leve & 8,2 & 7,0 & 6,0 & \\
\hline & & Moderado & 15,0 & 12,0 & 9,1 & \\
\hline & \multirow{2}{*}{$\begin{array}{l}\text { Qualidade } \\
\text { Vocal }^{\star \star}\end{array}$} & Adaptada & 5,1 & 5,0 & 3,1 & \multirow{2}{*}{$0,012^{*}$} \\
\hline & & Alterada & 10,0 & 8,0 & 7,2 & \\
\hline \multirow{5}{*}{ Desvantagem } & \multirow{3}{*}{$\mathrm{Grau}^{* \star *}$} & Sem Alteração & 2,6 & 3,0 & 2,0 & \multirow{3}{*}{0,136} \\
\hline & & Leve & 5,8 & 3,5 & 6,1 & \\
\hline & & Moderado & 7,2 & 4,0 & 6,6 & \\
\hline & \multirow{2}{*}{$\begin{array}{l}\text { Qualidade } \\
\text { Vocal }^{\star \star}\end{array}$} & Adaptada & 2,6 & 2,5 & 2,0 & \multirow{2}{*}{$0,049^{*}$} \\
\hline & & Alterada & 6,3 & 4,0 & 6,1 & \\
\hline \multirow{5}{*}{ Defeito } & \multirow{3}{*}{ Grau $^{* \star *}$} & Sem Alteração & 8,7 & 9,0 & 4,4 & \multirow{3}{*}{0,258} \\
\hline & & Leve & 11,4 & 11,0 & 7,2 & \\
\hline & & Moderado & 17,2 & 10,0 & 11,3 & \\
\hline & \multirow{2}{*}{$\begin{array}{l}\text { Qualidade } \\
\text { Vocal }^{\star *}\end{array}$} & Adaptada & 8,5 & 9,0 & 4,4 & \multirow{2}{*}{0,075} \\
\hline & & Alterada & 13,0 & 11,0 & 8,3 & \\
\hline \multirow{5}{*}{ Total } & \multirow{3}{*}{ Grau $^{* \star *}$} & Sem Alteração & 16,5 & 18,0 & 7,4 & \multirow{3}{*}{0,058} \\
\hline & & Leve & 25,3 & 22,5 & 17,4 & \\
\hline & & Moderado & 39,4 & 31,0 & 23,8 & \\
\hline & \multirow{2}{*}{$\begin{array}{l}\text { Qualidade } \\
\text { Vocal }^{\star \star}\end{array}$} & Adaptada & 16,1 & 17,0 & 7,4 & \multirow{2}{*}{$0,015^{\star}$} \\
\hline & & Alterada & 29,2 & 25,0 & 19,3 & \\
\hline
\end{tabular}

Teste ${ }^{* \star}$ Mann-Whitney e ${ }^{* \star *}$ Kruskal-Wallis ${ }^{*} p<0,05$

$\mathrm{Na}$ sequência, aparecem as subescalas incapacidade e desvantagem, respectivamente. Tais resultados corroboram dados obtidos por outros estudos $^{8-10}$. Estudo realizado com cantores de coros universitários de música popular brasileira obteve a informação de que tanto cantores com queixas quanto os sem queixas vocais apresentam maiores escores na subescala defeito, que corresponde ao domínio orgânico do IDV ${ }^{8}$. Tais resultados eram esperados e podem ser explicados por fatores como falta de domínio técnico, demanda vocal elevada na fala e limitada experiência no canto ${ }^{15}$.

Nossos achados mostram que os participantes que não realizaram aula de canto obtiveram maiores escores na subescala desvantagem do protocolo IDCM. Isso pode ser explicado devido ao provável uso incorreto da voz que pode prejudicar a saúde vocal do cantor amador, o que justifica a prática fonoaudiológica junto a este público ${ }^{1,5,16}$. Assim, queixas ou alterações na voz cantada podem ser decorrentes de vários fatores como a falta de conhecimentos específicos sobre aspectos relacionados à produção vocal, classificação vocal errônea, uso vocal incorreto e a não utilização de técnicas de aquecimento e desaquecimento vocais ${ }^{5,17}$. A prática de aulas de canto é fundamental para cantores e devem, de preferência, durar muitos anos. As aulas de canto promovem o desenvolvimento de todo o mecanismo vocal, o que contribui para uma melhor qualidade de voz cantada sem que ocorra esforço ${ }^{17,18}$. Estudos recentes têm apontado que os problemas vocais mais relatados pelos cantores podem decorrer da falta de técnica vocal ou pelas escassas orientações sobre bem-estar vocal ${ }^{5}$.

A ausência de diferença estatisticamente significante entre os escores médios das subescalas do IDCM e sexo, naipe e utilização da voz profissional falada, sugere que coralistas amadores de ambos os sexos e de diferentes naipes apresentam dificuldades vocais semelhantes. Tais variáveis, portanto, parecem não exercer influência sobre a desvantagem vocal autorreferida pelo grupo. Assim, é reforçada a idéia de que os problemas enfrentados pelos cantores estejam relacionados a outros fatores, tais como cuidado na classificação vocal realizada pelo regente, distribuição homogênea e semelhança nas exigências vocais nos diversos naipes ${ }^{10,13,15}$. 
Ao atribuírem uma nota de zero a dez à própria voz, observou-se que os cantores que se autoavaliaram com notas piores, obtiveram maiores escores referentes à desvantagem vocal no IDCM. A autoavaliação da voz pode complementar a análise vocal do indivíduo e deve ser associada à anamnese e à análise perceptivo-auditiva e computadorizada da voz, elucidando as possíveis etiologias dos distúrbios vocais ${ }^{15}$. Em nosso estudo, fica claro que por meio de uma simples atribuição de nota, feita pelo próprio cantor, obtiveram-se dados sobre o impacto de um possível problema de voz em sua vida e em suas atividades diárias.

Ao correlacionar os domínios idade, tempo de canto e carga horária de canto semanal com o protocolo IDCM, verificou-se que não houve diferença estatisticamente significante, ou seja, a desvantagem parece independer de tais variáveis. Em outro estudo, foi observado que não há relação entre a quantidade de sintomas vocais referidos por cantores e o tempo de atividade de canto ${ }^{5}$. Quanto à faixa etária, salienta-se que sujeitos acima de 59 anos foram excluídos da pesquisa, devido à possibilidade de apresentarem alterações vocais decorrentes da idade ${ }^{16}$. Tal critério pode ter contribuído para que a amostra fosse homogênea em relação a esta variável e, por isso, a diferença estatística não ocorresse.

Os cantores com vozes adaptadas autorreferiram menor desvantagem vocal, quando comparados aos que apresentaram vozes alteradas. Além disso, constatou-se que, quanto maior o grau de alteração, maior a desvantagem vocal, referente à incapacidade. Estudos apontam que indivíduos com queixas vocais duradouras apresentam maior desvantagem vocal ${ }^{19} \mathrm{e}$ indivíduos com distúrbios da voz apresentaram escores mais elevados na aplicação de protocolos ${ }^{12}$. Com isso, era esperado que cantores com queixas ou alterações de voz apresentassem maior desvantagem vocal em relação à coralistas sem tais queixas ${ }^{8}$.

Pesquisas evidenciam que grande parte dos cantores de Igreja não possui preparo vocal adequado e é raro que tenham acompanhamento fonoaudiológico. Isso contribui para que grande parte desta população apresente queixas em relação à voz cantada, como por exemplo, dificuldade para atingir determinados tons, cansaço vocal, pigarro, rouquidão, ardor ao cantar ${ }^{4,6}$. Muitas vezes, as orientações vocais que os coralistas de igreja recebem ocorrem de forma superficial ${ }^{3}$ e os problemas vocais mais relatados pelos indivíduos são referentes à falta de técnica vocal ${ }^{5}$.

A população de cantores é considerada de risco para desenvolvimento de problemas vocais. Por isso, conhecer os fatores que podem influenciar no desenvolvimento de uma disfonia facilitará a atuação dos fonoaudiólogos junto a esse grupo ${ }^{20}$. É importante que o fonoaudiólogo compreenda melhor as particularidades envolvidas no ato de cantar, essencialmente no que diz respeito aos níveis respiratório, fonatório, articulatório e ressonantal $^{1}$, para que possa desenvolver um trabalho adequado junto aos cantores. O fonoaudiólogo é considerado referência de orientação, quando comparado com os diferentes especialistas que se destinam a orientar pessoas que utilizam à voz profissionalmente ${ }^{21}$. É importante, portanto, que os fonoaudiólogos atentem-se às reais necessidades desses profissionais, para que possam desenvolver e implementar programas para promoção e prevenção específicos e assertivos ${ }^{22}$.

\section{CONCLUSÃO}

A partir dos resultados obtidos pode-se concluir que cantores de igreja apresentam desvantagem vocal importante, principalmente no que se refere às limitações orgânicas. Cantores com alterações vocais apresentam maiores desvantagem do que cantores com vozes adaptadas. Além disso, quanto maior o grau de alteração vocal, maiores as limitações referentes à voz cantada. A realização prévia de aulas de canto parece minimizar os efeitos referentes à desvantagem vocal. A desvantagem vocal neste grupo é semelhante entre homens e mulheres, independentemente da idade, naipe que cantam e associação de utilização da voz profissional falada. 


\begin{abstract}
Purpose: to evaluate the vocal handicap of amateur singers of church choirs. Method: we interviewed 42 amateur singers from church choirs, 20 men, and 22 women, between 18 and 59 year old. Everybody answered a questionnaire containing questions about self-perception of singing and vocal practices and the protocol Modern Singing Handicap Index (MSHI), composed by 30 questions regarding disability, handicap, and defect. We performed a screening for perceptual classification of adapted or changed voices, and measured the degrees of change. Results: the total average score was 23 points in the MSHI. The highest subscale scores obtained were "defect" (10.9), followed by "disability" (7.6) and "handicap" (4.5), with the difference between them $(p=0.001)$. Singers who have never passed through singing lesson had higher scores in the "handicap" $(p=0.003)$. The higher was the score of MSHI, the score given by singers in relation to their own voice sank $(p=0.046)$. Participants with altered voice quality had higher scores on handicap and disability subscales and on total domination of MSHI when compared with those who have adapted vocal quality $(p=0.012$, $p=0.049$ and $p=0.015$, respectively). Moreover, the greater is the degree of voice alteration, increased scores were related to disability subscale $(p=0.022)$. Conclusion: church singers have major vocal handicap. When you have voice disorders, this handicap is even greater. The higher is the degree of voice alteration, the greater are the limitations regarding the singing voice. Vocal singing lessons seem to minimize the handicap.
\end{abstract}

KEYWORDS: Voice; Voice Disorders; Habits; Music

\section{REFERÊNCIAS}

1. Andrade SR, Fontoura DR. Inter-relações entre fonoaudiologia e canto. Rev Acad Mus. 2007; 7(1): 83-98.

2. Silva MAA, Ferreira LP, Costa HO. Caracterização de um grupo de cantores da noite: um enfoque fonoaudiológico. ACTA ORL. 2008; 26 (4): 231-4.

3. Leite GC A, Assumpção R, Campiotto AR, Silva MAA. O canto nas igrejas: o estudo do uso vocal dos coralistas e não-coralistas. Dist Comum. 2004; 16(2): 229-39.

4. Costa PJBM, Ferreira KL, Camargo ZA, Pinho SMR. Extensão Vocal de Cantores de Coros Evangélicos Amador. Rev. CEFAC. 2006; 8(1): 96-106.

5. Ribeiro VV, Santos AB, Bonki E, Prestes $T$, Leite APD. Identificação de Problemas Vocais Enfrentados por Cantores de Igreja. Rev CEFAC. [No prelo]. 2010.

6. Behlau MS, Rehder MIBC. Perfil Vocal de Regentes de Coral do Estado de São Paulo. Rev. CEFAC. 2008; 10(1): 206-17.

7. Soares EB, Brito CMCP. Hábitos e perfil vocal em coralistas. ACTA ORL. 2009; 27(1): 28-35.

8. Moreti F, Rocha C, Borrego MCM, Behlau M. Desvantagem vocal no canto: análise do protocolo Índice de Desvantagem para o Canto Moderno IDCM. Rev Soc Bras Fonoaudiol. 2011; 16(2): 146-51.
9. Santos LMA, Gasparini G, Behlau M. Validação do protocolo do Índice de Desvantagem Vocal (IDV) no Brasil [monografia]. São Paulo: Centro de Estudos da Voz; 2007.

10. Ávila MEB, Oliveira G, Behlau M. Índice de desvantagem vocal no canto clássico (IDCC) em cantores eruditos. Pró-Fono. 2010; 22 (3): 221-6.

11. Murry T, Zschommler A, Prokop J. Voice handicap in singers. J Voice. 2009; 23(3): 376-9.

12. Behlau M, Santos LMA, Oliveira G. Crosscultural adaptation and validation of the voice handicap index into brazilian Portuguese. $\mathrm{J}$ Voice. 2009; 25(3): 354-9.

13. Rosen CA, Murry T. Voice handicap index in singers. Journal Voice. 2000; 14(3): 370-7.

14. Behlau M, Oliveira G, Santos LMA, Ricarte A. Validação no Brasil de protocolos de auto-avaliação do impacto de uma disfonia. Pró-Fono. 2009; 21(4): 326-32.

15. Jotz GP, Bramati O, Schimidt VB, Dornelles S, Gigante LP. Aplicação do "Voice Handicap Index" em coralistas. Arq ORL. 2002; 6(4): 260-4.

16. Menezes LN, Vicente LCC. Envelhecimento vocal em idosos institucionalizados. Rev CEFAC. 2007;9:90-8.

17. Dinville C. Os distúrbios da voz e sua reeducação. Rio de Janeiro: Enelivros; 2001.

18. Watts C, Barnes-Burroughs K, Estis J, Blanton $D$. The singing power ratio as an objective measure of singing voice quality in untrained talented and nontalented singers. J Voice. 2006; 20(1): 82-8. 
19. Cohen SM, MD, MPH, Noordzij JP, Garrett CG, Ossoff $\mathrm{RH}$. Factors associated with perception of singing voice handicap. Otolaryngology Head Neck Surg. April 2008; 138(4): 430-4.

20. Silva MA, Duprat AC. Voz cantada. In: Ferreira LP, Befi-Lopes DM, Limongi SCO. Tratado de Fonoaudiologia. São Paulo: Rocca; 2004. p.177-94.
21. Ueda KH, Santos LZ, Oliveira IB. 25 anos de cuidados com a voz profissional: avaliando ações. Rev. CEFAC. 2008; 10(4): 557-65.

22. Phyland DJ, Oates J, Greenwood KM. Selfreported voice problems among three groups of professional singers. J Voice. 1999; 13 (4): 602-11.

http://dx.doi.org/10.1590/S1516-18462012005000035

RECEBIDO EM: 18/08/2011

ACEITO EM: 12/10/2011

Endereço para correspondência:

Tatiane Prestes

Rua Pararaíba, 2034, Bairro dos Estados

Guarapuava - PR

CEP: 85035-190

E-mail: tatiane_prestes@yahoo.com.br 